\title{
Fluidic haptic interface for mechano-tactile feedback
}

\author{
G. Shi*, A. Palombi*, Z. Lim, A. Astolfi, A. Burani, S. Campagnini, F.G.C. Loizzo, M. Lo Preti, \\ A. Marin Vargas, E. Peperoni, C.M. Oddo, J. Hardwicke, M. Venus, S. Homer-Vanniasinkam, H.A. Wurdemann
}

\begin{abstract}
Notable advancements have been achieved in providing amputees with sensation through invasive and non-invasive haptic feedback systems such as mechano-, vibro-, electrotactile and hybrid systems. Purely mechanical-driven feedback approaches, however, have been little explored. In this paper, we now created a haptic feedback system that does not require any external power source (such as batteries) or other electronic components. The system is low-cost, lightweight, adaptable and robust against external impact (such as water). Hence, it will be sustainable in many aspects. We have made use of latest multimaterial 3D printing technology (Stratasys Objet500 Connex3) being able to fabricate a soft sensor and a mechano-tactile feedback actuator made of a rubber (TangoBlack Plus) and plastic (VeroClear) material. When forces are applied to the fingertip sensor, fluidic pressure inside the system acts on the membrane of the feedback actuator resulting in mechano-tactile sensation. We present the design, fabrication and validation of the proposed haptic feedback system. Our $\varnothing 7 \mathrm{~mm}$ feedback actuator is able to transmit a force range between $0.2 \mathrm{~N}$ (the median touch threshold) and $2.1 \mathrm{~N}$ (the maximum force transmitted by the feedback actuator at a $3 \mathrm{~mm}$ indentation) corresponding to force range exerted to the fingertip sensor of $1.2-18.49 \mathrm{~N}$.
\end{abstract}

\section{INTRODUCTION}

A CCORDING to the World Health Organization (WHO), 95\% of the 40 million amputees in developing countries lack access to affordable and appropriate prosthetic care and affordable devices [1]. Limb loss is disproportionately high in low and middle-income countries (LMICs) [1] which house $80 \%$ of the world's 3 million upper limb amputees. Some countries suffer the adverse legacy of previous conflicts, such as uncleared landmines in Sri Lanka; road accidents being considerably higher in LMICs [2]; and less stringent health and safety rules in factories compared to the developed world. Various illnesses can lead to limb loss: Examples include diabetes (the second largest contributor to loss of limbs after traffic accidents in Bangladesh [2]), polio, meningitis and stroke. Trauma accounts for the majority of upper limb amputations (31.5\%) [3]. In particular, partial-hand amputations due to work-related incidents are the most frequently amputated body parts [2]. The numbers of amputees are again disproportionally

*Both authors contributed equally to this manuscript.

G. Shi, A. Palombi, Z. Lim, S. Homer-Vanniasinkam, H.A. Wurdemann are with the Department of Mechanical Engineering, University College London, London, United Kingdom. E-mail: h.wurdemann@ucl.ac.uk.

A. Astolfi, A. Burani, S. Campagnini, F.G.C. Loizzo, M. Lo Preti, A. Marin Vargas, E. Peperoni, C.M. Oddo are with The BioRobotics Institute and Department of Excellence in Robotics and A.I., Scuola Superiore Sant'Anna, Pisa, Italy.

J. Hardwicke, M. Venus are with the University Hospitals Coventry and Warwickshire NHS Trust, Warwick, United Kingdom.

The research presented in this paper has been supported by the Grand Challenges Small Grant received from UCL Grand Challenges, the Royal Academy's of Engineering 2017/2018 Frontiers of Engineering for Development award and the Erasmus+ Traineeship programme by the EC.

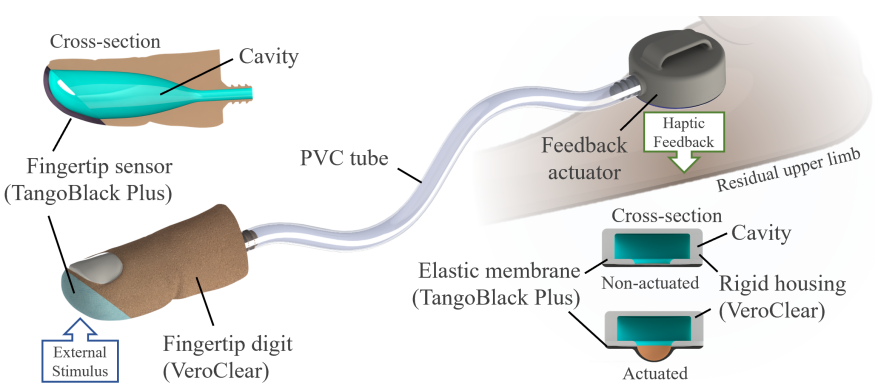

Fig. 1. CAD drawing of the haptic feedback system. The sensor/feedback actuator are made of 3D printed materials VeroClear and TangoBlack Plus and filled with water connected via a pipe. The feedback actuator can be mounted on the amputee's residual limb.

higher in newly-industrialised countries, primarily due to lower health and safety reinforcements. Depending on the severity, affected workers may require long-term, frequent intensive medical care (in fact, work-related injuries have the highest number days away from work [4]) as well as psychological support [5]. Despite cosmetic solutions for partial-hand amputees, currently available prosthetic solutions include bodyand battery-powered devices [4].

Body-powered prostheses are driven by the retained function of the next most proximal joint from the site of amputation (e.g., by the metacarpophalangeal joint flexion/extension or the wrist). In general, a tendon [6] or rigid mechanism [7] connects the prosthetic fingertip with a wristband. Activating the metacarpophalangeal joint results in a bending motion. On the other hand, battery-powered partial-hand prostheses embed electro-mechanical actuators (which can be controlled by (surface) Electromyography (EMG) interfaces). For instance, Shape Memory Alloys are integrated into an anthropomorphic finger: heating and cooling the flexor and extensor actuators cause the finger to flex and extend [8]. Other patented prosthetic fingers rely on servo-motors [9]. Extensive advancements have been achieved in providing the amputee with sensation through invasive and non-invasive haptic feedback systems. In [10], [11], mechano-, vibro-, electro-tactile and hybrid systems have been reviewed. Purely mechanical-driven feedback approaches, however, have been little explored. A prominent concept has been proposed by Rosset [12]. In 1933, he described how pressure at the prosthetic fingertip could be transmitted to amputees. In [13], this preliminary idea resulted in a pneumatic closed-loop haptic feedback system prototype which was validated by subjects. In 1953, Conzelman et al. were granted a patent on a similar haptic feedback system that used incompressible fluids instead of compressible air [14]. Technical challenges of the two aforementioned approaches include the liability (appearance of leakages) and fabrication 
process. Pneumatic transmission further has limitations due to its density and compressibility when effective displacement by only a few millilitre volume is required.

In this paper, we present the development and evaluation of a 3D printed mechano-tactile haptic feedback system using a hydraulic soft tactile fingertip sensor as shown in Figure 1. The contributions of our paper are as follows:

- Our haptic feedback system is purely mechanical. Compared to current systems relying on a mechatronic sensor/actuator system, our system is fundamentally different as it relies on a coupled hydraulic system.

- The proposed system is lightweight and low-cost as it is purely mechanical and does not require electrical components, e.g., batteries or a microcontroller.

- Our system can be manufactured using latest multimaterial additive technology only.

Latest technology in multi-material additive manufacturing such as the Stratasys Objet500 Connex3 allow printing of dual material such as VeroClear, a polymethyl methacrylate and TangoBlack Plus, a thermoplastic elastomer with flexible, rubber-like qualities. Our haptic feedback system is made of a TangoBlack Plus fingertip sensor integrated in a VeroClear finger linked to a wearable haptic mechano-tactile actuator with a TangoBlack Plus membrane. The sensor and actuator are 3D printed and can hence be easily integrated into (3D printed) body-powered upper limb prostheses. In case of distal amputations such as in the case of transradial amputees, the haptic feedback actuator can be interfaced with the residual limb/forearm through a socket. We report on engineering validation and results when used by healthy subjects.

Section II gives an overview of the entire haptic interface and describes the design process. In Section III, the fingertip sensor and feedback actuator are modelled. Engineering verification and validation with healthy subjects are conducted and critically assessed in Section IV. Section V summarises the key achievements and provides a look ahead.

\section{DESIGN OF THE HAPTIC FEEDBACK SYSTEM}

Our proposed concept in Figure 1 is a purely mechanically driven feedback system able to sense physical interactions with the environment and consequently transfer the tactile perception back to the user with mechano-tactile stimulation. The two key components include a fingertip sensor and a feedback actuator. A soft elastic membrane shapes the outer surface of a rigid fingertip with an inner cavity. A second elastic membrane is used for the base of the feedback actuator. A rigid housing with a cylindrical cavity is affixed on top of the elastic membrane. The two components are connected via a PVC hose to form a closed system.

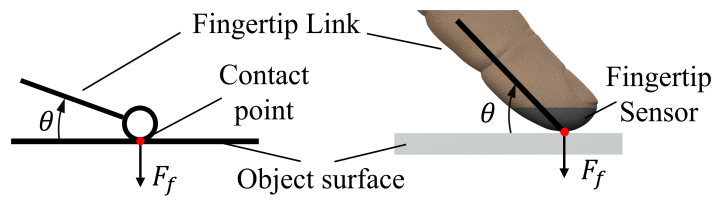

Fig. 2. Fingertip model when in contact with the surface of an object. The angle $\theta$ formed by fingertip link and object surface is depicted.
Water is used as the fluid medium inside the two cavities as well as the connecting hose. When a force is exerted to the elastic membrane of the fingertip, it deforms resulting in a decrease of the volume. The internal liquid pressure increases inside the closed system causing the membrane of the haptic feedback actuator to bulge and inflate. If the feedback actuator is worn by an end-user, the ballooning effect will exert a force on the contacted skin tissue and evoke a haptic sensation.

\section{A. Creating the fingertip sensor}

Our fingertip sensor is inspired by the design and deformation of a natural human fingertip, when interacting with objects, and modelled as a sphere [15] as shown in Figure 2. When grasping an object, the surface of the sphere forms a tangent at the contact point. The fingertip exerts a force $F_{f}$ perpendicular to the object resulting in friction which prevents slipping. The contact angle $\theta$ between the fingertip link and tangential surface of the object is under $60^{\circ}$ in stable grasping situations [16]. To allow homogeneous deformation and a stable grasp, the incline angle $\theta$ between the fingertip sensor and the fingertip link is chosen here to be $60^{\circ}$.

The overall design of our fingertip digit is based on the dimensions and curvature of a 24-year-old male's index fingertip which was 3D scanned by Structure Sensor (Occipital, Inc.) and imported into CAD software Solidworks (Dassault Systemes SE). The model was modified by generating the cavity and elastic fingertip membrane. The prototype was then printed with the rigid body made from VeroClear material and a $1 \mathrm{~mm}$ thick rubber-like membrane of TangoBlack Plus of 26 shore A hardness. The cavity inside the fingertip is $3.4 \mathrm{ml}$.

\section{B. Design of the feedback actuator}

A rigid housing has been created with an embedded cylindrical cavity. A $1 \mathrm{~mm}$ thick elastic membrane seals the actuator. Amplification can be achieved by varying the membrane diameter (see Figure 3(a) for diameters that were trialed here). Similar to the fingertip, the housing was printed using VeroClear material whereas TangoBlack Plus was used for the membrane. The fingertip sensor and haptic feedback actuator are connected via a $3 \mathrm{~mm}$ hose. Increase of the internal pressure due to fingertip deformation results in a ballooning effect as shown in Figures 3(b) and (c): when the actuator is placed on the human's skin, the swelling of the membrane produces an indentation, hence, mechano-tactile stimulation.
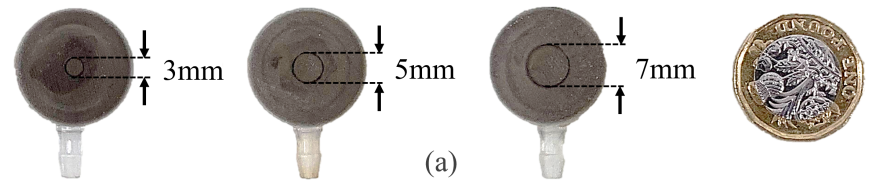

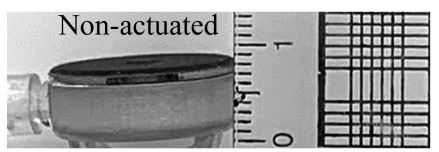

(b)

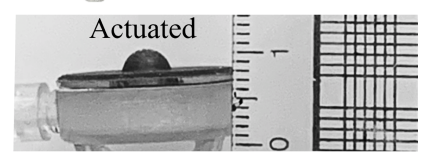

(c)
Fig. 3. (a) Feedback actuator prototypes with various membrane diameters (Pound coin for reference). Actuation example of a feedback actuator (b) without and (c) with stimulus on the fingertip. 


\section{Finite Element Model OF THE FINGERTIP SENSOR}

The membrane of the fingertip sensor has a convex irregular shape made from TangoBlack Plus, a non-linear elastic material. To understand deformation limitations during physical interaction and the relationship between deformation and reaction force during indentation, a Finite Element Model (FEM) analysis was executed in ANSYS.

Firstly, ten $1 \mathrm{~mm}$ thick TangoBlack Plus specimens were subjected to uniaxial tensile tests, according to international standard protocols [17]. In the FEM simulation, the first order Mooney-Rivlin model was used (see Equation 1) to fit the experimental stress-strain curves.

$$
U=C_{10}\left(I_{1}-3\right)+C_{01}\left(I_{2}-3\right)+\frac{1}{D_{1}}(J-1)^{2}
$$

where $U$ is the strain energy, $I_{1}$ and $I_{2}$ are the first and second invariant of the left Cauchy-Green deformation tensor, $J$ is the elastic volume strain, $C_{10}$ and $C_{01}$ are the material constants and $D_{1}$ is the impressibility parameter. This hyperelastic model is suitable to mathematically describe TangoBlack Plus as the maximum strain here is lower than $30 \%$ [18]. The material constants were adjusted to fit the experiments by minimising the mean squared error resulting in the following values: $C_{01}=0.033 \mathrm{MPa}, C_{10}=1.271 \mathrm{MPa}$ and $D_{1}=$ $1.08 \mathrm{MPa}$. Then, the geometry of the fingertip was imported to ANSYS from Solidworks defining the aforementioned values for the materials. A number of assumptions and hypotheses were made to reduce the computational cost:

- The fingertip membrane was analysed in the FEM.

- The indentation direction was parallel to the fingertip section to inspect the maximum deformation and stress distribution as shown in Figure 4(a).

- Progressive indentation was analysed to allow simulation of multiple steps in case the convergence failed.

The setup of the simulation is shown in Figure 4. A rigid plate was placed under the fingertip with the contact surface being parallel to the fingertip section. The plate then moved towards the fingertip causing a $3 \mathrm{~mm}$ indentation (see Figure 4(b)). The result of the FEM are shown in Figure 5. After the fingertip was indented, the contact surface between the rigid plate and fingertip sensor became flat with no irregular distortion. The maximum deformation was $3.2 \mathrm{~mm}$, located at the most prominent point of fingertip. The total deformation of each element reduced from the maximum deformation point to the rim of fingertip sensor.

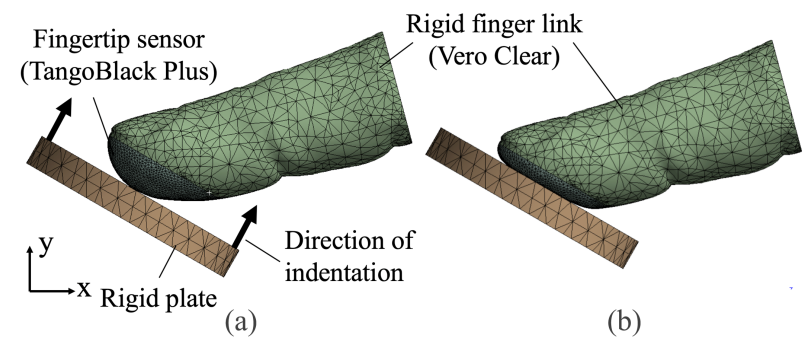

Fig. 4. FEM setup of the fingertip sensor in Ansys (a) before indentation and (b) when indented by $3 \mathrm{~mm}$.
The FEM simulation can inspect the structure of fingertip sensor during indentation and predict failure of the material behaviour, using Von-Mises failure criterion theory. The boundary condition can be defined in Equation 2.

$$
\sigma^{\prime} \geq S_{y}
$$

where $S_{y}$ is tensile yield strength and $\sigma^{\prime}$ is Von-Mises stress. The maximum Von-Mises equivalent stress was $3.782 \mathrm{MPa}$ on the inner surface. In Figure 5, the result of the FEM shows maximum Von-Mises stresses of 3.782 MPa in the inner surface of the fingertip sensor exceeding the tensile strength of TangoBlack Plus material. A cross sectional view shows that only the surface layer exceeds the tensile strength rather than the stress penetrating the entire thickness of the membrane. Hence, this area of the fingertip membrane might be at risk of failure caused by higher stress values according the FEM.

\section{VERIFICATION AND VALIDATION OF MECHANICAL-BASED HAPTIC FEEDBACK SYSTEM}

\section{A. Experiment 1: Characterising the haptic feedback system}

Protocol: This experiment was carried out to determine the physical relationship between the fingertip and the elastic membrane of feedback actuator as well as to compare the performance of three feedback actuators with different diameters of the elastic membrane (see Figure 3(a)). The FEM simulation was first validated by testing the physical limitations of the fingertip sensor without the feedback actuator and the water medium. Following this, the force and internal liquid pressure of the complete closed-loop haptic system was also tested, analysing the force exerted and internal liquid pressure at both the fingertip sensor and feedback actuator. Hence, the fingertip sensor was indented $3 \mathrm{~mm}$ at a speed of $5.81 \mathrm{~mm} / \mathrm{s}$, at a perpendicular direction to the tangential surface of the fingertip sensor. Data related to the force exerted to the fingertip sensor and feedback actuator as well as internal hydraulic pressure. Each trial was carried out five times for each actuator and an average was taken across the trials.

Experimental setup: An experimental workbench was constructed as shown in Figure 6, consisting of a liner rail (Zaber X-LSM100A) with $0.05 \mu \mathrm{m}$ sensitivity, a 3-axis force sensor (IIT-FT17) with $0.318 * 10^{-3} \mathrm{~N}$ sensitivity, a fluidic pressure transducer (OMEGA PXM319-001G) with $0.05 \mathrm{kPa}$ sensitivity and a load cell (Honeywell-FSAXX001RC4C5) with $0.22 * 10^{-3} \mathrm{~N}$ sensitivity. The force sensor was placed opposite the fingertip sensor to ensure that the fingertip would be homogeneously pressed. The linear rail drove the force sensor towards the fingertip sensor to stimulate the haptic

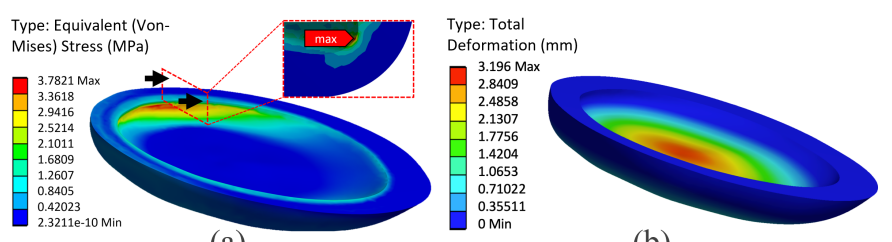

(a)

(b)

Fig. 5. FEM simulation result of fingertip sensor: (a) Von-Mises stress values. (b) Total deformation from isometric point of view. 
feedback system and the indentation and reaction force were recorded at the fingertip sensor. In addition, pressure change was recorded from the pressure transducer and the load cell recorded the force from the feedback actuator.

Test result: The results of the FEM simulation compared with the experimental results (Experimental 1) are shown in Figure 7(a). The loading curves both show similar non-linear curves, where the results for the reaction force in Experiment 1 increases at a faster rate than the FEM simulation. The maximum reaction force at the fingertip sensor was $5.84 \mathrm{~N}$ in Experiment 1 compared to $4.88 \mathrm{~N}$ in the FEM simulation, which occurred when the indentation was $3 \mathrm{~mm}$.

Each of the results for the tests relating to the reaction force and internal liquid pressure of the complete closedloop system are shown in Figures 7(b)-(d). In each graph, the $\varnothing 3 \mathrm{~mm}$ actuator is represented by the blue line, $\varnothing 5 \mathrm{~mm}$ in yellow dashed, and $\varnothing 7 \mathrm{~mm}$ is shown by the red line. The graph displaying the force against indentation displacement is shown in Figure 7(b). Each feedback actuator produced similar non-linear curves with hysteresis values of $19.9 \%, 18.6 \%$ and $20.5 \%$ corresponding to the order $\varnothing 3 \mathrm{~mm}, \varnothing 5 \mathrm{~mm}$ and $\varnothing 7 \mathrm{~mm}$. When the fingertip sensor had a maximum indentation of $3 \mathrm{~mm}$, the reaction forces for the $\varnothing 3 \mathrm{~mm}, \varnothing 5 \mathrm{~mm}$ and $\varnothing 7 \mathrm{~mm}$ were $16.97 \mathrm{~N}, 16.95 \mathrm{Nand} 18.49 \mathrm{~N}$ respectively.

Figure 7(c) shows the relationship between the internal liquid pressure and indentation for each sized feedback actuator. These results also produced non-linear curves with hysteresis values of $18.6 \%, 17.0 \%$ and $17.2 \%$ corresponding to $\varnothing 3 \mathrm{~mm}$, $\varnothing 5 \mathrm{~mm}$ and $\varnothing 7 \mathrm{~mm}$. At the maximum indentation, the internal liquid pressure was $38.01 \mathrm{kPa}, 37.92 \mathrm{kPa}$ and $38.11 \mathrm{kPa}$, each corresponding to $\varnothing 3 \mathrm{~mm}, \varnothing 5 \mathrm{~mm}$ and $\varnothing 7 \mathrm{~mm}$.

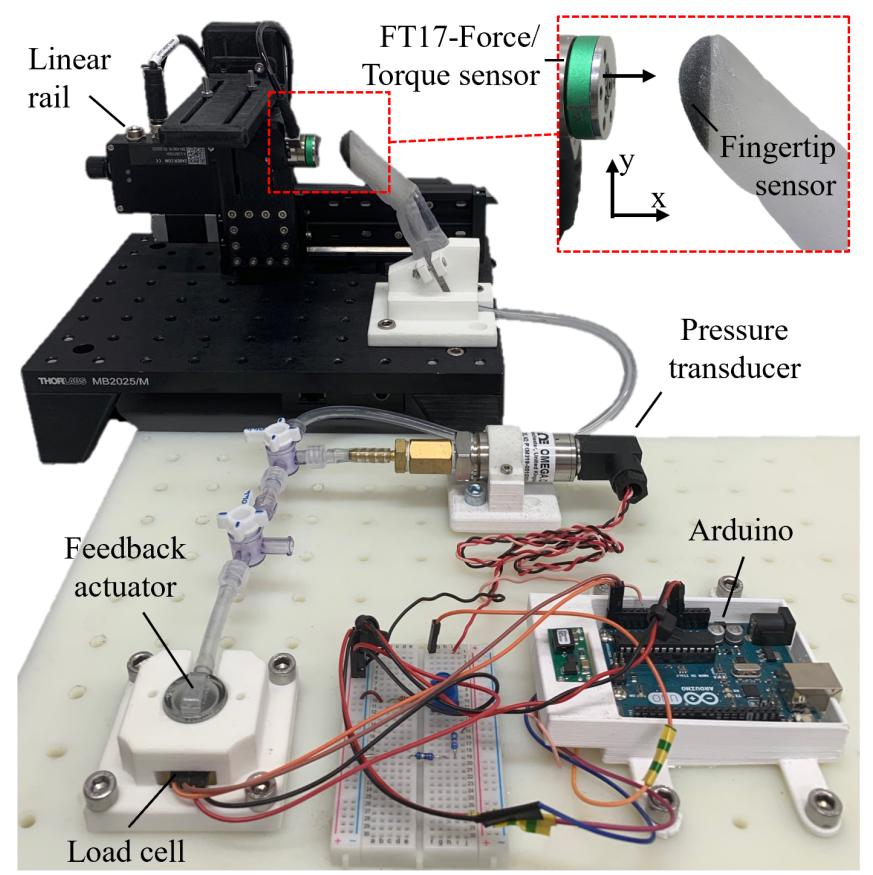

Fig. 6. Experimental setup: A Force/Torque sensor fixed to a linear rail opposes the fingertip sensor, indenting the sensor. Force readings are taken, an additional load cell measures the force from the feedback actuator. A pressure transducer monitors pressure change.
It was observed that the maximum hysteresis point for both the internal liquid pressure and force both occurred when the indentation was $2.2 \pm 0.1 \mathrm{~mm}$. Hence, the increase of reaction force and internal liquid pressure during indentation acts synchronously.

When the fingertip sensor is indented, the internal liquid pressure increases, causing the water medium to transfer the pressure increase to the feedback actuator. The elastic membrane in the feedback actuator then inflates/swells. The force exerted to the membrane of the feedback actuator is expressed in Equation 3.

$$
F_{a}=\left(\Delta P_{l}-\Delta P_{\eta}\right) * A_{m}
$$

where $\Delta P_{l}$ is the pressure increase, $\Delta P_{\eta}$ is the viscous pressure drop of water flow and $A_{m}$ is the effective contact area of the elastic membrane of feedback actuator. The viscous pressure drop $\Delta P_{\eta}$ is presented in Equation 4.

$$
\Delta P_{\eta}=f_{D} \cdot \frac{\rho}{2} \cdot \frac{\langle v\rangle^{2}}{D} \cdot L
$$

where $f_{D}$ is Darcy friction factor, $\rho$ is density of fluid, $\langle v\rangle$ is the mean flow velocity and $L$ the pipe length. The Darcy friction factor is determined by the Zigrang \& Sylvester's equation in Equation 5 [19].

$$
\begin{gathered}
\frac{1}{\sqrt{f_{D}}}=-2 \lg \left(\frac{\varepsilon / D}{3.7}-\frac{5.02}{R_{e}} \lg \left(\frac{\varepsilon / D}{3.7}+\frac{12}{R_{e}}\right)\right) \\
R_{e}=\frac{\rho Q D}{\mu A}
\end{gathered}
$$

where $\varepsilon$ is the relative roughness of PVC pipe as $0.0015, D$ is the diameter of the pipe, $Q$ is the flow rate, $A$ is the cross area of the pipe and $\mu$ is the viscosity of the water. Computing Equations 4-6, the pressure drop caused by water flowing from the fingertip sensor to the feedback actuator is approximately $1.2 \mathrm{kPa}$. During the experiments, a load cell measured the reaction force from the feedback actuator as a result of pressure increase. After compensating the pressure drop at the feedback actuator, the relationship between the internal liquid pressure and force from feedback actuator is linear, as shown in Figure 7(d). The $\varnothing 7 \mathrm{~mm}$ membrane produces the highest value for the maximum transmitted force, which is $2.1 \mathrm{~N}$ compared to $1.82 \mathrm{~N}$ from the $\varnothing 5 \mathrm{~mm}$ membrane, and $0.48 \mathrm{~N}$ from the $\varnothing 3 \mathrm{~mm}$ membrane. The $\varnothing 3 \mathrm{~mm}$ membrane is less capable of transmitting the force producing a slope of $0.99 \mathrm{~N} / \mathrm{kPa}$ compared to the larger membranes, as a relatively high pressure is required to overcome the surface tension changing from a flat into a convex configuration. Hence, the $\varnothing 3 \mathrm{~mm}$ membrane was abandoned in Experiment 2.

\section{B. Experiment 2: Human interaction tests}

Protocol: This experiment was designed to determine the stimulus threshold of the feedback actuator as a means to observe how users responded to the haptic feedback system. This study has been approved by UCL Research Ethics Committee (under application number 12453/001). A number of participants individually validated the feedback actuators with $\varnothing 5 \mathrm{~mm}$ and $\varnothing 7 \mathrm{~mm}$ membranes as these two haptic feedback 


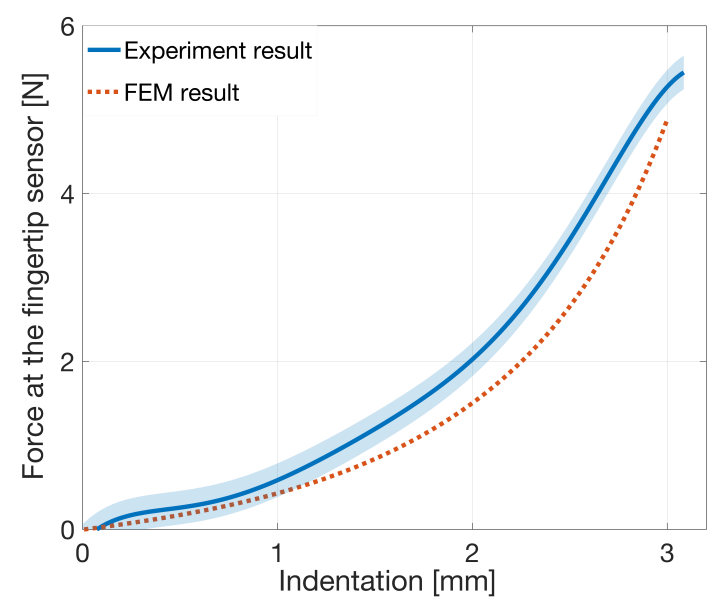

(a)

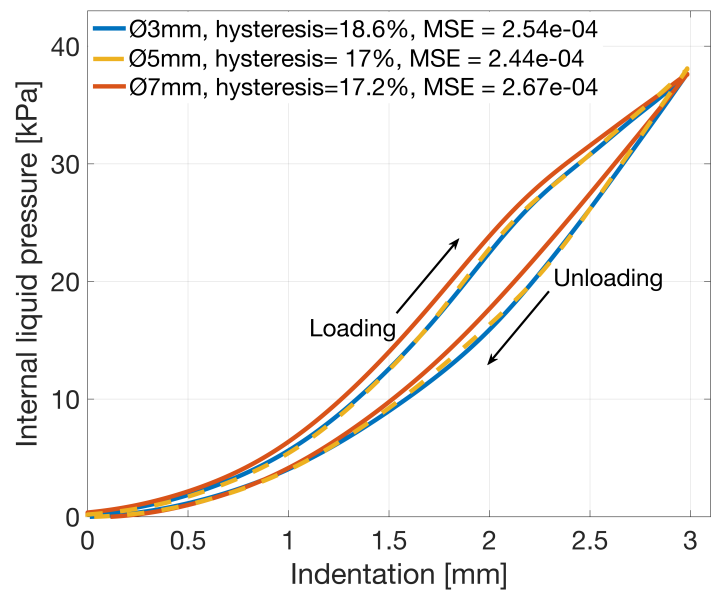

(c)

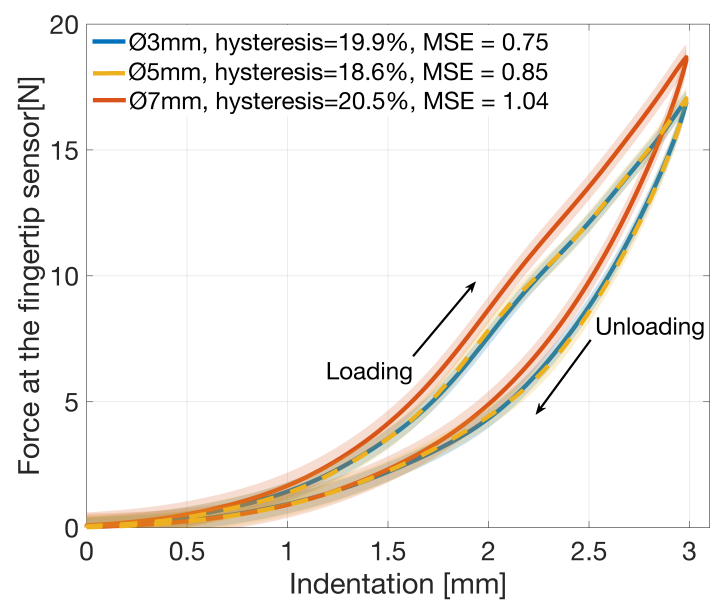

(b)

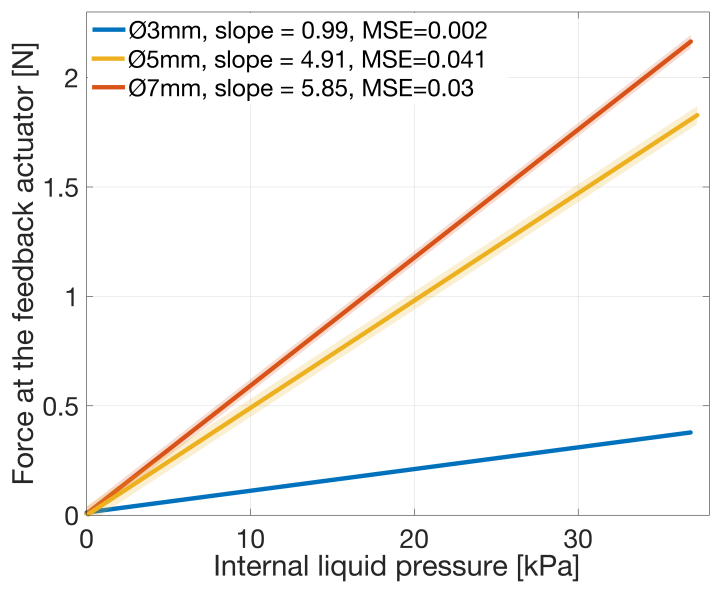

(d)

Fig. 7. (a) Non-linear reaction force of the fingertip sensor against indentation: The predicted results from the FEM simulation and experimental test are plotted. (b) Non-linear loading and unloading reaction force curves against indentation. (c) Non-linear internal liquid pressure against indentation for the full closed-loop feedback system comparing $\varnothing 3 \mathrm{~mm}, \varnothing 5 \mathrm{~mm}$ and $\varnothing 7 \mathrm{~mm}$ membranes. (d) Linear relationships between the output force at the feedback actuator and the internal liquid pressure for each feedback actuator membrane.

actuators outperformed the $\varnothing 3 \mathrm{~mm}$ membrane with respect to feedback force range (see Figure $7(\mathrm{~d})$ ). All participants attended a trial with 50 stimuli. In each stimulus, the force of the feedback actuator worn was applied at a fixed value ranging between $0.1-1.0 \mathrm{~N}$ (in $0.1 \mathrm{~N}$ increments), and held at this level for $1 \mathrm{~s}$. Following each stimulus, the participant confirmed if they could or could not identify the haptic sensation. The threshold for each participant was recorded.

Test setup: 10 participants were sat on a chair and asked to place their forearm in a comfortable resting position on a table. Vision and auditory senses were obscured to reduce the potential effects of external disturbances. The feedback actuator was then strapped to the upper side of the participant's forearm, $10 \mathrm{~cm}$ from their wrist. Varying stimuli were generated by the force sensor applying different indentations on the fingertip sensor, which was fixed to the workbench as in Experiment 1. Due to the linear relationship between the force at feedback actuator and internal pressure obtained in Experiment 1, the force at feedback actuator was accurately controlled by the internal liquid pressure during the fingertip indentation.

Test result: The results of the 10 participants testing both the $\varnothing 5 \mathrm{~mm}$ and $\varnothing 7 \mathrm{~mm}$ feedback actuators are shown in Table 1. During the stimulus threshold test, the relationship between the force stimulation of feedback actuator and reaction force at fingertip sensor followed the same relationship observed in the hardware test. In the test data, the median touch threshold result for the $\varnothing 5 \mathrm{~mm}$ feedback actuator is $0.2 \mathrm{~N}$ with an interquartile range (IQR) of $0.175 \mathrm{~N}$. For the $\varnothing 7 \mathrm{~mm}$ feedback actuator, the median touch threshold is $0.2 \mathrm{~N}$ with an IQR of $0.1 \mathrm{~N}$ describing the statistical dispersion.

\section{Discussion}

In Experiment 1, our fingertip sensor produced a reaction force of $10.44 \mathrm{~N}$ when it was indented by $2.3 \mathrm{~mm}$ at a $60^{\circ}$ contact angle. In [20], when a natural fingertip was indented 
TABLE I: Stimulus threshold test results of $\varnothing 5 \mathrm{~mm}$ and $\varnothing 7 \mathrm{~mm}$.

\begin{tabular}{c|c|c} 
Gender/Age & $F_{f} / F_{s}(\varnothing 5 \mathrm{~mm})$ & $F_{f} / F_{s}(\varnothing 7 \mathrm{~mm})$ \\
\hline $\mathrm{F} / 22$ & $0.5 \mathrm{~N} / 2.9 \mathrm{~N}$ & $0.1 \mathrm{~N} / 0.6 \mathrm{~N}$ \\
$\mathrm{~F} / 22$ & $0.2 \mathrm{~N} / 1.2 \mathrm{~N}$ & $0.2 \mathrm{~N} / 1.22 \mathrm{~N}$ \\
$\mathrm{M} / 21$ & $0.1 \mathrm{~N} / 0.58 \mathrm{~N}$ & $0.2 \mathrm{~N} / 1.22 \mathrm{~N}$ \\
$\mathrm{M} / 23$ & $0.1 \mathrm{~N} / 0.58 \mathrm{~N}$ & $0.2 \mathrm{~N} / 1.22 \mathrm{~N}$ \\
$\mathrm{M} / 24$ & $0.5 \mathrm{~N} / 2.9 \mathrm{~N}$ & $0.7 \mathrm{~N} / 4.5 \mathrm{~N}$ \\
$\mathrm{M} / 33$ & $0.3 \mathrm{~N} / 1.8 \mathrm{~N}$ & $0.2 \mathrm{~N} / 1.22 \mathrm{~N}$ \\
$\mathrm{~F} / 23$ & $0.2 \mathrm{~N} / 1.2 \mathrm{~N}$ & $0.2 \mathrm{~N} / 1.22 \mathrm{~N}$ \\
$\mathrm{M} / 28$ & $0.2 \mathrm{~N} / 1.2 \mathrm{~N}$ & $0.2 \mathrm{~N} / 1.22 \mathrm{~N}$ \\
$\mathrm{~F} / 20$ & $0.3 \mathrm{~N} / 1.8 \mathrm{~N}$ & $0.3 \mathrm{~N} / 1.8 \mathrm{~N}$ \\
$\mathrm{M} / 26$ & $0.2 \mathrm{~N} / 1.2 \mathrm{~N}$ & $0.3 \mathrm{~N} / 1.8 \mathrm{~N}$
\end{tabular}

${ }^{*}$ M: Male, F: Female, $F_{f}$ : Force of feedback actuator, $F_{s}$ : Force stimuli on the fingertip.

under the same conditions, it produced a reaction force of $7 \mathrm{~N}$. Both studies produced similar non-linear indentationforce curves, and so it can be concluded that our fingertip sensor is slightly stiffer than a natural human fingertip.

In Experiment 2, when two feedback actuators were tested with a $\varnothing 5 \mathrm{~mm}$ and $\varnothing 7 \mathrm{~mm}$ membrane, the $\varnothing 7 \mathrm{~mm}$ feedback actuator exerted a larger stimulus range between $0.2 \mathrm{~N}$ (the median touch threshold) and $2.1 \mathrm{~N}$ (the maximum force transmitted by the feedback actuator at a $3 \mathrm{~mm}$ indentation as shown in Figure 7(d)). The corresponding force range exerted on the sensor was $1.2-18.49 \mathrm{~N}$ as shown in Figure 7(b). Although the haptic feedback system provides a modalitymatching tactile stimulation, the deviation between the reaction force and force at feedback actuator still exists and might not completely restore the nature in-born haptic sensation.

Although the touch threshold median of the $\varnothing 5 \mathrm{~mm}$ and $\varnothing 7 \mathrm{~mm}$ diameters were the same, the $\varnothing 7 \mathrm{~mm}$ feedback actuator ensures a more consistent performance compared to the $\varnothing 5 \mathrm{~mm}$ as evident by the smaller IQR of $0.1 \mathrm{~N}$ : Analysing the results from Table 1, the stimulus threshold detected by each participant is slightly higher when the $\varnothing 7 \mathrm{~mm}$ membrane was used. This can be attributed to the increase of contact area between the user's skin and feedback actuator membrane by $75.4 \mathrm{~mm}^{2}$, resulting in a larger stimulation area with a lower indentation that is detected consistently compared to a smaller stimulation with a higher indentation force.

\section{Conclusions}

In this paper, we have developed a novel purely mechanicalbased haptic feedback system. It can sense the force applied to a fingertip sensor and feed back to an actuator to generate tactile stimuli. The design of the fingertip sensor and feedback actuator features TangoBlack Plus in both the fingertip and membrane of the feedback actuator. Both components are linked via a tube filled with a hydraulic medium to transfer the pressure between the fingertip and the feedback actuator.

Verification and validation tests were performed. Output forces of the fingertip were compared to an FEM to determine the limitations and the non-linear relationship between deformation and reaction force during indentation. Further analysis of the whole system included measuring the reaction force at both the fingertip sensor and feedback actuator in addition to the internal liquid pressure for three different feedback actuators with $\varnothing 3 \mathrm{~mm}, \varnothing 5 \mathrm{~mm}$ and $\varnothing 7 \mathrm{~mm}$ membrane diameters. In each case, the force at the fingertip sensor and internal liquid pressure produced similar loading and unloading curves with little difference in hysteresis. However, the relationship between the force of the feedback actuator and the internal liquid pressure showed considerable differences, where the $\varnothing 3 \mathrm{~mm}$ produced a much lower force. On the other hand, a human interaction test was conducted: Users wore the feedback actuator and were stimulated at varying forces to determine the stimulus threshold of the feedback actuators with $\varnothing 5 \mathrm{~mm}$ and $\varnothing 7 \mathrm{~mm}$ diameter membranes. It was observed that while the median stimulus threshold for both actuators was $0.2 \mathrm{~N}$, the $\varnothing 7 \mathrm{~mm}$ membrane produced a more consistent performance across the sample of participants.

In the future, we are interested in how vibration can be fed back and processed to the end user. Furthermore, the haptic feedback system will be integrated into body-powered prostheses and tested with amputees with full vision and auditory senses.

\section{REFERENCES}

[1] WHO, Guidelines for training personnel in developing countries for prosthetics/orthotics services, 2005

[2] WHO, Global status report on road safety, 2015.

[3] British Society of Rehabilitation Medicine, "Amputee and Prosthetic Rehabilitation - Standards and Guidelines", 3rd Edition; Report of the Working Party (Co-Chairs: R.S. Hanspal, I. Sedki), London 2018.

[4] I. Imbinto, C. Peccia, M. Controzzi, A.G. Cutti, A. Davalli, R. Sacchetti, C. Cipriani, "Treatment of the Partial Hand Amputation: An Engineering Perspective," IEEE Reviews Bio-Med Eng, vol. 9:32-48, 2016.

[5] E.M. Price, K. Fisher, "How does counselling help people with amputation?" J Prosthet Orthot, vol. 14: 102-106, 2002.

[6] B.D. Veatch, "Joint and digit," US Patent 15276753, 2016

[7] Partial Hand Solutions LLC., "Prosthetic Fingers," Available on http://www.partialhandsolutions.com/products.html. [Accessed: Dec. 8, 2018].

[8] E. Engeberg, S. Dilibal, M. Vatani, J. Choi, J. Lavery, "Anthropomorphic finger antagonistically actuated by SMA plates," Bioinspir. Biomim. vol. 10(5):1-15, 2015.

[9] M. Haslinger, "Gripping device," US Patent 8915528, 2014

[10] J.S. Schofield, K.R. Evans, J.P. Carey, J.S. Hebert, "Applications of sensory feedback in motorized upper extremity prosthesis: A review," Expert Rev. Med. Devices, vol. 11(5):1-13, 2014.

[11] P. Svenssona, U. Wijkb, A. Björkmanb, C. Antfolka, "A review of invasive and non-invasive sensory feedback in upper limb prostheses," Expert Rev. Med. Devices, vol. 14(6):439-447, 2017.

[12] D.S. Childress, "Closed-loop control in prosthetic systems: Historical perspective," Ann. Biomed. Eng., vol. 8:293-303, 1980.

[13] C. Antfolk, A. Björkman, S.-O. Frank, F. Sebelius, G. Lundborg, B. Rosen, "Sensory feedback from a prosthetic hand based on air mediated pressure from the hand to the forearm skin," J. Reha- bil. Med., vol. 44:702-707, 2012

[14] J. E. Conzelman, H.B. Ellis, C.W. O’Brien. US Patent 2,656,545, Prosthetic Device Sensory Attachment, 1953.

[15] C. Borst, M. Fischer, G. Hirzinger, "Calculating hand configurations for precision and pinch grasps," IEEE/RSJ Int. Conf. on Intell. Robots and Systems, pp. 1553-1559, 2002.

[16] R. Murray, Z. Li, and S. Sastry, A mathematical introduction to robotic manipulation. CRC Press, 1994.

[17] BS, 'Rubber, Vulcanized or Thermoplastic_-Determination of the Effect of Liquids," British Standards Institution, London, UK, Standard No. BS ISO 1817, 2015.

[18] J.E. Ryu, E. Salcedo, H.J. Lee, S.J. Jang, E.Y. Jang, H. Al Yassi, D. Baek, D. Choi, E. Lee, "Material models and finite analysis of additively printed polymer composites,' Journal of Composite Materials, vol. 53(3):361-371,2018.

[19] S. Genic, I. Arandelovic, P. Kolendic, M. Jaric, N. Budimir, V. Genic, "A review of explicit approximations of Colebrook's equation", FME Transactions, 39(2):67-71, 2011.

[20] H. Han, S. Kawamura, "Analysis of stiffness of human fingertip and comparison with artificial fingers," IEEE Int. Conf. on Systems, Man, and Cybernetics, pp. 800-805, 1999. 